\title{
Construction of Motion Model of Motion Simulation System of Airport Fire Engine Simulator
}

\author{
Lishan Jia ${ }^{1,2, a}$, Liwen Wang ${ }^{1,2, b}$ \\ ${ }^{1}$ Tianjin Key Laboratory for Civil Aircraft Airworthiness and Maintenance of Civil Aviation University \\ of China, Civil Aviation University of China, Tianjin 300300, China; \\ ${ }^{2}$ Ground Support Equipments Research Base of Civil University of China, Civil Aviation University of \\ China, Tianjin 300300, China \\ ajlshfd163@yeah.net, ,wlw2885@163.com
}

Keywords: simplified motion model of vehicle; fire engine simulator; motion simulation system; real-time; fidelity

\begin{abstract}
To the high real-time quality requirement of motion simulation system of airport fire engine simulator, a simulation system with simplified motion model was promoted. The simplified motion model simplifies motion model of vehicle based on application requirement of virtual training of vehicle driving. On one hand, the simplification can reduce calculation amount of simulation, on the other hand, the simplification can make it easier to get parameters of model. Motion simulation system based on simplified motion model of airport fire engine was constructed and experiment was taken. Experiment showed that the motion simulation system has high calculation speed and good fidelity through setting parameters properly.
\end{abstract}

\section{Introduction}

The fire safety of airport has always been the focus of attention. According to the International Civil Aviation Organization (ICAO) Statistics, 71\% of the crashed aircraft caught fire and burned in the accident at the airport ${ }^{[1]}$. So it is the top priority to control the fire of the airport security. Airport fire engine drivers need higher skill than ordinary fire engine drivers because of the special working environment of the airport fire engines. Airport fire engine simulator is a simulation device designed specially for training airport fire engine driver. Motion simulation system is one of the sub system of airport fire engine simulator, which calculates the motion status parameters of vehicle according to input of driver and transmits them to other physical effect systems (audio, visual, instrument) of simulator.

Construction of motion model of automobile by analyzing working theory is a complex problem. Literature [2] described the interaction and transmission of forces of the engine, the clutch, the gearing, the brake, and the wheels during the driving or braking or shifting process. And a vehicle motion model was given through the dynamics of rigid-body analysis of the vehicle. Literature [3] gave a vehicle motion model of 14-degree of freedom using in real-time simulation. Literature [4] used neural-adaptive output feedback control on wheeled inverted pendulum models of vehicle. Literature [5] promoted a new vehicle motion model for automotive active safety systems of automobile. But on the whole, the motion models mentioned above are complex and need a lot of parameters of vehicle that might be hard to get. And which might influence real-time performance and fidelity of simulation system.

Airport fire engine simulator is a virtual training device of human in the loop type. The simulator emphasizes real-time performance and fidelity, but would not care about if the motion model is based on dynamics analysis of vehicle body. So a simplified vehicle motion model was constructed. The motion model could decrease the calculation amount of simulation system, and could make output of model be the same as real vehicle through proper setting of parameters.

\section{Composition and principle of the airport fire engine simulator}


The airport fire engine simulator is composed of hardware part and software part. Hardware part include: the simulator cockpit devices, computer systems, visual display system and the interface device. Software part include: control system, airport fire engine motion simulation system, visual system, sound system, instructor system and data transmission control. The schematic of airport fire truck simulator is shown in Figure 1. Instructor system will transmit the information of training programs to the fire engine motion simulation system and the visual system. Control system collects control data from system driver, fire engines motion simulation system calculates vehicle state parameters based on the firefighter input and passes it to other systems. Visual system displays the virtual scene based on vehicle location, speed, acceleration, and other information in real time. Instrument system displays the current state of the vehicle. Sound system generates sounds of the vehicle working. Firefighters experience the virtual environment provided by these systems through vision, hearing, feeling and make the appropriate input into the training simulator. This creates the interactive environment of one person per car so that firefighters can be trained.

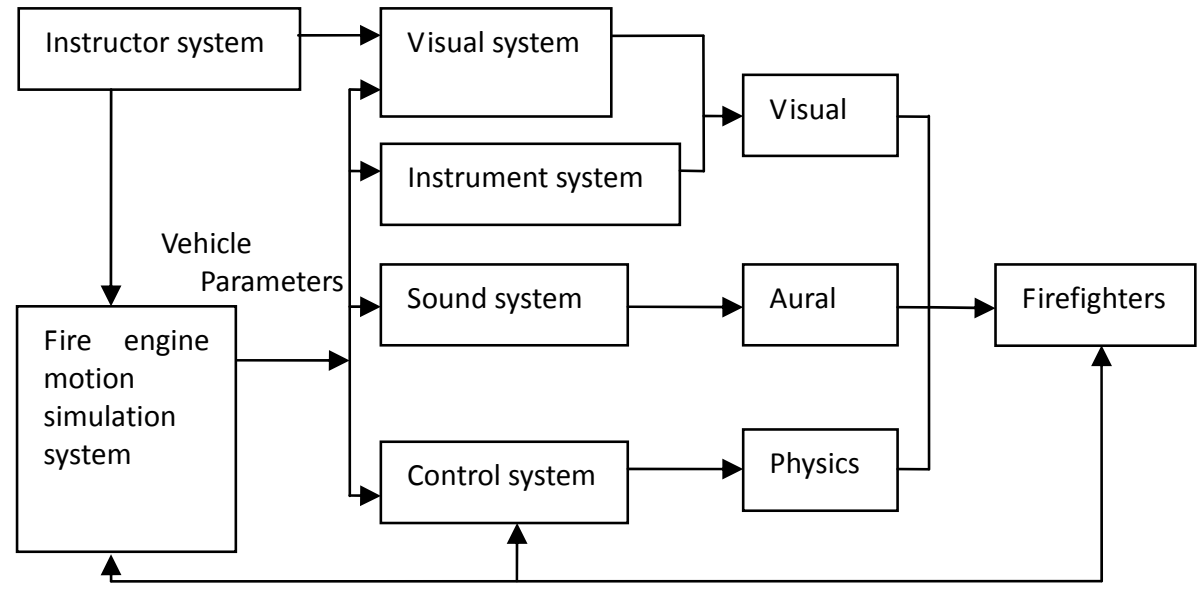

Fig. 1. Airport fire engine simulator principle

\section{Simulation of vehicle}

Normally, vehicle dynamics includes engine mechanics, motor resistance mechanics, transmission system mechanics, braking system mechanics, suspension system mechanics and steering system mechanics. The motion of vehicle is a acceleration changing motion. The changing of acceleration or speed lies on the dynamic mechanics of the vehicle.

The engine simulation relates to the relationship between rotation speed of engine and output torque. When throttle is $100 \%$, the relationship could be expressed as full characteristic curve. When throttle is lower than $100 \%$, the relationship could be expressed as part characteristic curve. And the rotation speed could be gotten from vehicle speed and max output torque. The motor resistance includes air resistance, roll resistance, grade resistance. The resistance could be gotten from force analyzing. Vehicle transmission system includes clutch, transmission, universal transmission shaft and drive bridge. The mechanics of transmission system could be gotten through research of the mechanics of these sub systems. There are two kinds of braking force in braking system, ground braking force and brake braking force. The function of braking of vehicle comes from the working of the two forces. But the getting of each of the two forces need to take forces analyzing and experience summarize. In vehicle simulation, the suspending system could be seen as spring system connecting vehicle body and vehicle wheels. Through shape changing of spring, the change of relative position of body and wheels could be simulated. Steering system is generally composed of by three parts of the steering operation agencies, steering gear and steering linkage. Steering system is a certain stiffness when at work, ignoring the steering stiffness simplifies it into a fully rigid system.

It can be seen from the statements above that motion model of vehicle constructed from working principle analyzing is complex and has a lot of parameters being hard to gotten, which might influence the real-time performance and fidelity of simulation system. Airport fire engine simulator 
is a virtual training simulation system, which emphasizes the real-time performance and fidelity but not whether it is based on the working principle. So the motion model could be simplified to show the outside performance of fire engine that is enough for training of firefighters.

\section{Simplified motion model of fire engine}

Take the ground coordinate system of visual system as coordinate system. Let origin of coordinate be $O$, axis $Z$ pass the origin $O$ and point to the sky, axis $Y$ pass the origin $O$ and point to the direction of zero heading angle, axis $X$ pass the origin $O$ and point to the vertical direction to the plane of $Y O Z$.

Let the velocity of vehicle be $V$, coordinate be $(x, y, z)$, heading angle be $\phi$. Initial position of vehicle is

$\left\{\begin{array}{l}x=x_{0} \\ y=y_{0} \\ z=z_{0}\end{array}\right.$

Initial heading angle is

$\phi=\phi_{0}$

Initial velocity is

$V=V_{0}$

Change of heading angle by control is $\Delta \phi$, then

$\phi=\phi+\Delta \phi$

Change of coordinate is $(\Delta x, \Delta y, \Delta z)$, let step of simulation be $h$, then

$\left\{\begin{array}{l}\Delta x=V^{*} \sin \phi^{*} h \\ \Delta y=V^{*} \cos \phi^{*} h \\ \Delta z=0\end{array}\right.$

$\left\{\begin{array}{l}x=x+\Delta x \\ y=y+\Delta y \\ z=z+\Delta z\end{array}\right.$

Let the throttle value be $t(t \in[0,1])$, let the max increase value of velocity be $\Delta V_{i \max }$, then increase value of velocity to throttle value $\Delta V_{i}$ is

$\Delta V_{i}=t \Delta V_{i \max }$

Let the brake value be $b(b \in[0,1])$, let the max decrease value of velocity be $\Delta V_{d \max }$, then decrease value of velocity to brake value $\Delta V_{d}$ is

$\Delta V_{d}=b \Delta V_{d \max }$

Let decrease value of velocity owing to friction be $\Delta V_{f}$, Then change of velocity is

$\Delta V=\Delta V_{i}-\Delta V_{d}-\Delta V_{f}$

Then velocity of vehicle is 


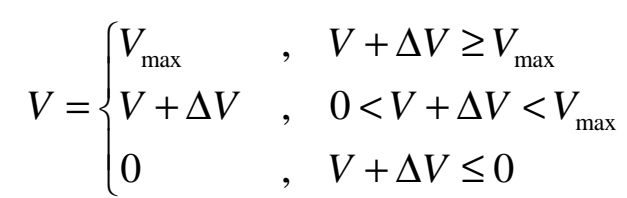

Let the max change of heading angle be $\Delta \phi_{\max }$, let the ratio of roll angle of wheel be $w(w \in[-1,1])$, then

$$
\Delta \phi=w \Delta \phi_{\max }
$$

Because the fire engine to be simulated has auto gear-box, the change of grade is not considered for sake of simplification.

The motion of pitching, rolling and motion in axis $Z$ are not included in the simplified motion model, because they could be gotten in visual system using collision detection technology. Literature [6] described a realization method of vehicle movement simulation in visual driving software Vega using collision detection technology. This arrangement can promote the real-time performance of training simulator.

The parameters of the simplified motion model are listed in table 1.

\begin{tabular}{|c|c|c|c|c|c|}
\hline \multicolumn{2}{|c|}{ Initial value } & \multicolumn{2}{|c|}{ Control parameter } & \multicolumn{2}{|c|}{ Input value } \\
\hline parameter & illustration & parameter & illustration & parameter & illustration \\
\hline \multirow{3}{*}{$\left(x_{0}, y_{0}, z_{0}\right)$} & \multirow{3}{*}{ initial coordinate } & $\Delta V_{i \max }$ & $\begin{array}{l}\text { the max increase } \\
\text { value of velocity }\end{array}$ & $h$ & $\begin{array}{c}\text { step of } \\
\text { simulation }\end{array}$ \\
\hline & & $\Delta V_{d \max }$ & $\begin{array}{l}\text { the max } \\
\text { decrease value } \\
\text { of velocity }\end{array}$ & $t(t \in[0,1])$ & $\begin{array}{l}\text { the throttle } \\
\text { value }\end{array}$ \\
\hline & & $\Delta V_{f}$ & $\begin{array}{l}\text { decrease value } \\
\text { of velocity } \\
\text { owing to friction }\end{array}$ & $b(b \in[0,1])$ & the brake value \\
\hline \multirow[b]{2}{*}{$\varphi_{0}$} & \multirow{2}{*}{$\begin{array}{l}\text { initial heading } \\
\text { angle }\end{array}$} & $V_{\max }$ & the max velocity & \multirow{2}{*}{$w(w \in[-1,1])$} & \multirow{2}{*}{$\begin{array}{l}\text { the ratio of roll } \\
\text { angle of wheel }\end{array}$} \\
\hline & & $\Delta \phi_{\max }$ & $\begin{array}{l}\text { the max change } \\
\text { of heading angle }\end{array}$ & & \\
\hline
\end{tabular}

Table 1 Parameters of simplified motion model

It can be seen from table 1 that the parameters are easy to get from driving test or experience. So the fidelity of fire engine motion simulation system can be satisfied.

Figure 2 shows the change of running distance according to change of velocity when throttle is full and grade is " $\mathrm{D}$ ”.

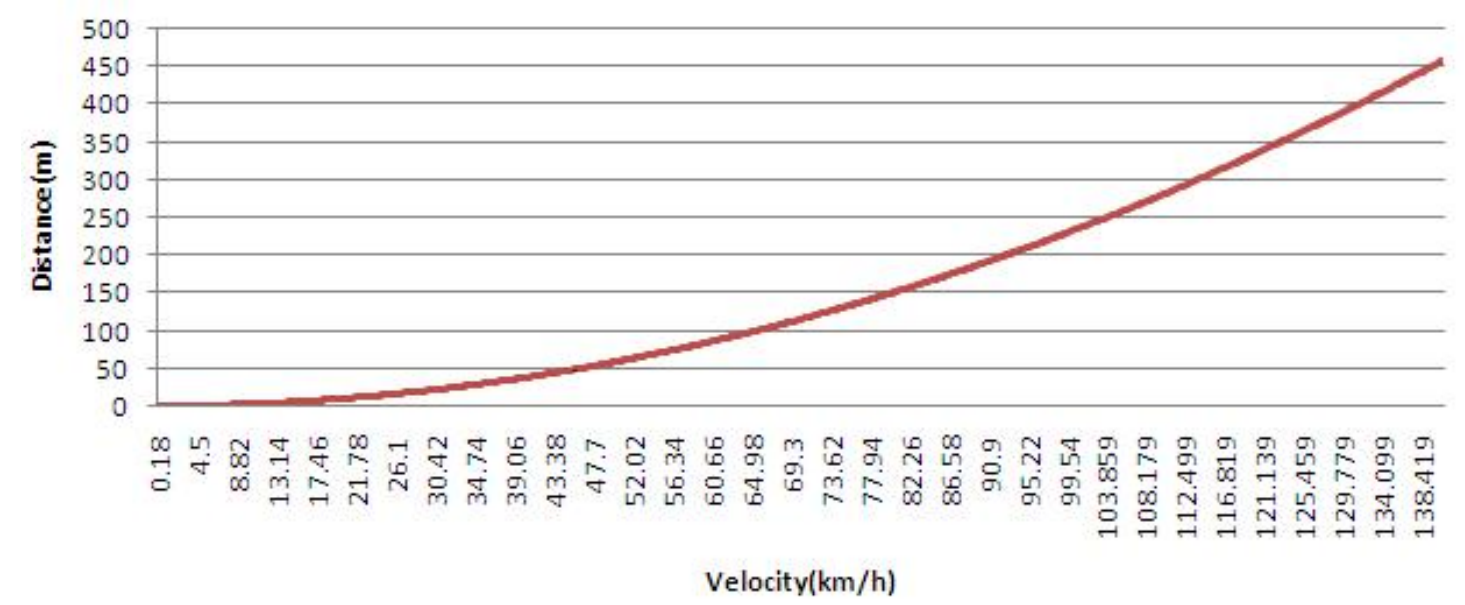

Fig. 2. Diagram of result of running of simplified motion model

\section{Summary}

The simplified motion model of airport fire engine has advantages of low calculation amount and easy to get parameters. Low calculation amount can satisfy the requirement of real-time 
performance of training system. The parameters of motion model can be gotten through driving test or just the experience of fire engine driver, which assures the fidelity of simulation of vehicle motion.

\section{Reference}

[1] Feng He, Songlin Dong. Basic performance requirements for airport fire engines. Fire Technique and Products Information, 3(3), 61-62(2004). (in Chinese)

[2] Yifeng Wang. Vehicle Dynamics Analysis and Simulation. Hangzhou: College of Computer Science of Zhejiang University, 2007. (in Chinese)

[3] Thomas Uchida, John McPhee. Driving simulator with double-wishbone suspension using efficient block-triangularized kinematic equations. Multibody Syst. Dyn. 28, 331-347 (2012).

[4] Zhijun Li, Chenguang Yang. Neural-Adaptive Output Feedback Control of a Class of Transportation Vehicles Based on Wheeled Inverted Pendulum Models. IEEE TRANSACTIONS ON CONTROL SYSTEMS TECHNOLOGY. 20(6), 1583-1591 (2012).

[5] Joakim Sörstedt, Lennart Svensson, and so on. A New Vehicle Motion Model for Improved Predictions and Situation Assessment. IEEE TRANSACTIONS ON INTELLIGENT TRANSPORTATION SYSTEMS. 12(4), 1209-1219 (2011).

[6] Peng Jiang, Zhengjun Zhai, Xue Li, and so on. Research and Realization of Vehicle Movement Simulation in Vega. Computer Simulation. 24(7), 268-271 (2007). (in Chinese) 